PROCEEDINGS OF THE

AMERICAN MATHEMATICAL SOCIETY

Volume 127, Number 5, Pages 1363-1370

S 0002-9939(99)04842-X

Article electronically published on January 28, 1999

\title{
HYPERINVARIANT SUBSPACES OF OPERATORS WITH NON-VANISHING ORBITS
}

\author{
LÁSZLÓ KÉRCHY
}

(Communicated by David R. Larson)

\begin{abstract}
It is shown that if the Banach space operator $T$ has regular normsequence, its vector orbits are asymptotically non-vanishing and there exists a complete vector orbit satisfying the growth condition of non-quasianalycity, then $T$ has infinitely many disjoint hyperinvariant subspaces.
\end{abstract}

In the recent paper [7] we initiated the study of operators possessing regular norm-sequences. The definition was based on the concept of almost convergence. We recall that a bounded, complex sequence $\xi \in \ell^{\infty}(\mathbf{N}, \mathbf{C})$ almost converges to the complex number $c$ if $\lim _{k \rightarrow \infty} \sup _{n \in \mathbf{N}}\left|c-k^{-1} \sum_{j=n}^{n+k-1} \xi(j)\right|=0$. (The reader is referred to [9] for fundamental properties of this type of convergence.) We say that the sequence $\xi$ almost converges to $c$ in the strong sense if $|\xi-c \mathbf{1}|$ almost converges to zero, where 1 stands for the constant 1 sequence. A gauge function is a mapping $p: \mathbf{N} \rightarrow(0, \infty)$ with the property that $\{p(n+1) / p(n)\}_{n \in \mathbf{N}}$ almost converges in the strong sense to a positive number $c$. The set of all gauge functions is denoted by $\mathcal{P}$.

Let us consider now a complex Banach space $\mathcal{X}$ and let $\mathcal{L}(\mathcal{X})$ denote the set of bounded, linear operators acting on $\mathcal{X}$. We say that the norm-sequence of an operator $T \in \mathcal{L}(\mathcal{X})$ is compatible with the gauge function $p \in \mathcal{P}$, if $\left\|T^{n}\right\| \leq p(n)$ holds for every $n \in \mathbf{N}$ and the sequence $\left\{\left\|T^{n}\right\| / p(n)\right\}_{n \in \mathbf{N}}$ does not almost converge to zero. The set of all such operators is denoted by $\mathcal{L}(p, \mathcal{X})$, and $T \in \mathcal{X}$ is said to have a regular norm-sequence if $T$ belongs to $\mathcal{L}(p, \mathcal{X})$ for some $p \in \mathcal{P}$. It was shown in [7] that $\{p(n+1) / p(n)\}_{n \in \mathbf{N}}$ almost converges to the spectral radius $r(T)$ for every $T \in \mathcal{L}(p, \mathcal{X})$, and that a large class of operators with positive spectral radius possesses regular norm-sequence, including power bounded operators of spectral radius one. With regard to the asymptotic behaviour of vector orbits the following subclasses can be introduced. The operator $T \in \mathcal{L}(p, \mathcal{X})$ belongs to the class $\mathcal{C}_{1} \cdot(p, \mathcal{X}) \quad\left(\mathcal{C}_{*} \cdot(p, \mathcal{X})\right)$ if $\left\{\left\|T^{n} x\right\| / p(n)\right\}_{n \in \mathbf{N}}$ does not almost converge to zero for all non-zero vectors $x \in \mathcal{X}$ (for some non-zero vector $x \in \mathcal{X}$, respectively).

In the present paper we are concerned with the hyperinvariant subspace lattice Hlat $(T)$ of operators $T$ of class $\mathcal{C}_{1} \cdot(p, \mathcal{X})$ and $\mathcal{C}_{*} \cdot(p, \mathcal{X})$. We remind the reader that a (closed) subspace $\mathcal{M}$ is hyperinvariant for $T$, and so it belongs to $\operatorname{Hlat}(T)$, if $C \mathcal{M} \subset$ $\mathcal{M}$ holds for every operator $C$ commuting with $T$. In [7] we proved among other things the existence of a proper hyperinvariant subspace under different conditions,

Received by the editors August 6, 1997.

1991 Mathematics Subject Classification. Primary 47A15, 47A60.

Research partially supported by Hungarian NFS Research grant no. T 022920.

(C)1999 American Mathematical Society 
extending former results due to B. Sz.-Nagy-C. Foias and A. Atzmon. Continuing these investigations we are going to prove now the following theorem, exploring structural properties of Hlat $(T)$ for a new class of operators with regular normsequences.

Theorem. Let $T \in \mathcal{L}(\mathcal{X})$ be an operator belonging to the class $\mathcal{C}_{1} .(p, \mathcal{X}), p \in \mathcal{P}$. Let us assume that there exists a sequence $\left\{x_{n}\right\}_{n \in \mathbf{Z}}$ in $\mathcal{X}$ such that the vectors $\left\{x_{n}\right\}_{n \in \mathbf{N}}$ span an infinite dimensional subspace, $T x_{n}=x_{n+1}$ holds for every $n \in \mathbf{Z}$, and

$$
\sum_{n \in \mathbf{Z}} \frac{\log ^{*}\left(r(T)^{-n}\left\|x_{n}\right\|\right)}{1+n^{2}}<\infty .
$$

Then there exists a sequence $\left\{\mathcal{X}_{n}\right\}_{n \in \mathbf{N}}$ of non-zero hyperinvariant subspaces of T such that

$$
\mathcal{X}_{n} \cap\left(\bigvee_{j \neq n} \mathcal{X}_{j}\right)=\{0\}
$$

holds for every $n \in \mathbf{N}$. Furthermore, if $\sigma_{p}(T) \cap r(T) \mathbf{T}=\emptyset$, then

$$
\bigcap_{n \in \mathbf{N}}\left(\bigvee_{j \geq n} \mathcal{X}_{j}\right)=\{0\}
$$

Here $\sigma_{p}(T)$ stands for the point spectrum (set of eigenvalues) of $T, \mathbf{T}$ denotes the unit circle $\{z \in \mathbf{C}:|z|=1\}$ in the complex plane $\mathbf{C}, \mathbf{Z}$ denotes the set of integers and $\mathbf{N}$ is the set of positive integers. Furthermore, $\log ^{*} t:=0$ if $0 \leq t \leq 1$ and $\log ^{*} t:=\log t$ if $t \geq 1$.

The previous result generalizes [2, Theorem XII.8.1], due to B. Beauzamy, in two respects. First, Beauzamy's theorem treats the special case when the gauge function $p$ is constant 1 . Second, our result claims the existence of infinitely many disjoint hyperinvariant subspaces, not merely the existence of a proper hyperinvariant subspace. We note that the vector $x_{0}$ itself can be cyclic for the commutant of $T$; take the bilateral shift on $\ell^{2}(\mathbf{Z})$ and choose the vector $x_{0}$ with only one non-zero coordinate. We remind the reader also of the fact that there are non-scalar operators which do not have disjoint hyperinvariant subspaces. For example, any two non-zero hyperinvariant subspaces of the unilateral shift (of arbitrary multiplicity) intersects in an infinite dimensional subspace.

Though our proof follows the outline of that of Beauzamy, several essential improvements are needed. In order to make our argument transparent we split the reasoning into a series of lemmas. Let us assume that the operator $T \in \mathcal{C}_{1} \cdot(p, \mathcal{X})(p \in$ $\mathcal{P})$ satisfies the conditions of the Theorem and that the spectral radius $r(T)=1$.

We know by [7, Theorem 1] that there exists an isometry $V_{+}$acting on a Banach space $\mathcal{Y}_{+}$and an injective transformation $X \in \mathcal{L}\left(\mathcal{X}, \mathcal{Y}_{+}\right)$with dense range such that $V_{+} X=X T$. Furthermore, for every operator $C$ in the commutant $\{T\}^{\prime}:=$ $\{A \in \mathcal{L}(\mathcal{X}): A T=T A\}$ there exists a unique operator $D_{+} \in\left\{V_{+}\right\}^{\prime}$ such that $D_{+} X=X C$. It may happen that the isometry $V_{+}$is not invertible. However, in view of [4] there always exists an invertible isometry $V$ acting on a larger space $\mathcal{Y}$ such that $V \mathcal{Y}_{+} \subset \mathcal{Y}_{+}, V \mid \mathcal{Y}_{+}=V_{+}, \mathcal{Y}=\bigvee_{n \in \mathbf{N}} V^{-n} \mathcal{Y}_{+}$and for every $D_{+} \in\left\{V_{+}\right\}^{\prime}$ there exists a unique $D \in\{V\}^{\prime}$ with the properties $D \mathcal{Y}_{+} \subset \mathcal{Y}_{+}$and $D \mid \mathcal{Y}_{+}=D_{+}$. The transformation $X$ can be considered as an injective element of $\mathcal{L}(\mathcal{X}, \mathcal{Y})$. This associated invertible isometry $V$ will be extensively used to get information for the operator $T$. 
A natural functional calculus can be developed for $V$. Indeed, let us consider the Banach algebra

$$
A(\mathbf{T}):=\left\{f \in C(\mathbf{T}):\|f\|:=\sum_{n \in \mathbf{Z}}|\hat{f}(n)|<\infty\right\}
$$

of continuous functions with absolutely convergent Fourier series. Since $\left\|V^{n}\right\|=1$ holds for every $n \in \mathbf{Z}$, we can define the operator $f(V):=\sum_{n \in \mathbf{Z}} \hat{f}(n) V^{n}$ for every $f \in A(\mathbf{T})$. It is clear that the mapping $\Psi_{V}: A(\mathbf{T}) \rightarrow \mathcal{L}(\mathcal{Y}), f \mapsto f(V)$ is a contractive algebra-homomorphism, $\chi^{n} \mapsto V^{n}(n \in \mathbf{Z})$, and that $\operatorname{ran} \Psi_{V}$ is contained in the bicommutant $\{V\}^{\prime \prime}$ of $V$. We shall exploit several known properties of the function algebra $A(\mathbf{T})$. To start with, let us mention that this algebra is normal, that is given any disjoint closed subsets $E$ and $F$ of the circle $\mathbf{T}$ there exists a function $f \in A(\mathbf{T})$ such that $f \mid E=1$ and $f \mid F=0$. Let $E$ be a nonempty closed subset of $\mathbf{T}$, let $k(E)$ denote the ideal of functions vanishing on $E$, and let us consider the quotient algebra $A(\mathbf{T}) / k(E)$. It is not difficult to show that every non-zero complex homomorphism of $A(\mathbf{T}) / k(E)$ is of the form $\delta_{\lambda}(\pi(f))=$ $f(\lambda)(f \in A(\mathbf{T}))$, where $\lambda \in E$ and $\pi: A(\mathbf{T}) \rightarrow A(\mathbf{T}) / k(E)$ is the quotient mapping. Therefore, we obtain that the spectrum $\sigma(\pi(f))$ coincides with the set $f(E)$, for every $f \in A(\mathbf{T})$. (For a thorough investigation of $A(\mathbf{T})$ we refer to [5] and [6].)

Now we attach a hyperinvariant subspace of $T$ to every function $f \in A(\mathbf{T})$.

Definition. Given any function $f \in A(\mathbf{T})$ let $\mathcal{X}(T, f)$ denote the subspace $\operatorname{ker}(f(V) X)$.

Lemma 1. The subspace $\mathcal{X}(T, f)$ is hyperinvariant for $T$, for every $f \in A(\mathbf{T})$.

Proof. Let $C \in\{T\}^{\prime}$ and $x \in \mathcal{X}(T, f)$ be arbitrary. There exists a unique $D \in\{V\}^{\prime}$ such that $D X=X C$. Thus, we have $f(V) X C x=f(V) D X x=D f(V) X x=$ $D 0=0$, and so $C x \in \mathcal{X}(T, f)$. Q.E.D.

The main point in what follows is to find functions in such a way that the corresponding subspaces be non-trivial (different from $\{0\}$ and $\mathcal{X}$ ) and disjoint. The second lemma concerns the relation of the induced hyperinvariant subspaces. For any function $f \in A(\mathbf{T})$, ker $f$ denotes the zero set of the function $f$ and $\operatorname{supp} f:=$ $(\mathbf{T} \backslash \operatorname{ker} f)^{-}$is the support of $f$.

Lemma 2. Given any functions $f, g \in A(\mathbf{T})$ the following implications are true:

(i) if ker $f \cap \operatorname{ker} g=\emptyset$, then $\mathcal{X}(T, f) \cap \mathcal{X}(T, g)=\{0\}$;

(ii) if ker $f$ is contained in the interior of $\operatorname{ker} g$, then $\mathcal{X}(T, f) \subset \mathcal{X}(T, g)$.

Proof. Let us suppose that ker $f \cap \operatorname{ker} g=\emptyset$, and let us consider a vector $u \in$ $\mathcal{X}(T, f) \cap \mathcal{X}(T, g)$. Then $f(V) X u=g(V) X u=0$. Since the function $h=\bar{f} f+\bar{g} g \in$ $A(\mathbf{T})$ has no zeros, we infer by the Paley-Wiener Theorem that the function $1 / h$ belongs to $A(\mathbf{T})$, and so the operator $h(V)$ is invertible. Thus the product $h(V) X$ is injective, and the equality $h(V) X u=\bar{f}(V) f(V) X u+\bar{g}(V) g(V) X u=0$ implies that $u=0$.

Let us assume now that $\operatorname{ker} f$ is contained in the interior $(\operatorname{ker} g)^{(0)}$ of $\operatorname{ker} g$. It follows that the function $f$ is not zero on the closed set $E=\mathbf{T} \backslash(\operatorname{ker} g)^{(0)}$, and clearly supp $g \subset E$. Considering the quotient algebra $A(\mathbf{T}) / k(E)$ we obtain that the spectrum $\sigma(\pi(f))=f(E)$ does not contain the zero. Hence $\pi(f)$ is invertible, that is there exists a function $h \in A(\mathbf{T})$ such that $\pi(f) \pi(h)=\pi(1)$. Since $f h-1 \in k(E)$ 
we infer that $g=f h g$. The equation $g(V)=f(V) h(V) g(V)$ readily implies that ker $f(V)$ is included in $\operatorname{ker} g(V)$. Q.E.D.

We proceed with giving sufficient condition for the relation $f(V) y \neq 0$ in terms of the local spectrum $\sigma(V, y)$. We note that the isometry $V$ obviously possesses the single-valued extension property, and that $\sigma(V, y)$ is a non-empty closed subset of the spectrum $\sigma(V) \subset \mathbf{T}$ for every non-zero vector $y \in \mathcal{Y}$. (For the fundamentals of local spectral theory we refer to [3].)

Lemma 3. Let us consider a non-zero vector $y \in \mathcal{Y}$ and a function $\varphi \in A(\mathbf{T})$. If there exists a point $z_{0} \in \sigma(V, y)$ such that $\varphi\left(z_{0}\right) \neq 0$, then $\varphi(V) y \neq 0$.

Proof. There exists an open $\operatorname{arc} \alpha$ on $\mathbf{T}$ containing $z_{0}$ such that $\varphi(z) \neq 0$ for every $z \in \alpha$. Let us consider the principal ideal $J=\{\varphi g: g \in A(\mathbf{T})\}$. Repeating the argument applied in the proof of the previous lemma, we obtain that $J$ contains every function $h \in A(\mathbf{T})$ with the property $\operatorname{supp} h \subset \alpha$.

Let us suppose that $\varphi(V) y=0$. It follows that $h(V) y=0$ whenever $\operatorname{supp} h \subset$ $\alpha, h \in A(\mathbf{T})$. Let us choose now an open arc $\alpha_{0}$ containing $z_{0}$ such that the closure $\alpha_{0}^{-}$is included in $\alpha$, and let us consider the closed set $E_{0}=\mathbf{T} \backslash \alpha_{0}$. For every function $h \in k\left(E_{0}\right)$ we have $\operatorname{supp} h \subset \alpha_{0}^{-} \subset \alpha$, and so $h(V) y=0$. Consequently, $\operatorname{ker} \Psi_{y} \supset k\left(E_{0}\right)$ is valid for the mapping $\Psi_{y}: A(\mathbf{T}) \rightarrow \mathcal{Y}, g \mapsto g(V) y$; hence we can form the quotient mapping $\tilde{\Psi}_{y}: A(\mathbf{T}) / k\left(E_{0}\right) \rightarrow \mathcal{Y}, \pi(g) \mapsto g(V) y$. Let $M$ denote the operator of multiplication by the identical function $\chi(z)=z(z \in \mathbf{T})$ acting on the Banach space $A(\mathbf{T})$, and let us consider the quotient operator $\tilde{M}=M / k\left(E_{0}\right)$. It is easy to check that $\tilde{\Psi}_{y} \tilde{M}=V \tilde{\Psi}_{y}$ and $\sigma(\tilde{M})=\sigma(\pi(\chi))=E_{0}$. Let us consider the analytic function $F(z)=\tilde{\Psi}_{y}(\tilde{M}-z I)^{-1} \pi(1)$ defined on $\mathbf{C} \backslash E_{0}$. Since $(V-z I) F(z)=$ $(V-z I) \tilde{\Psi}_{y}(\tilde{M}-z I)^{-1} \pi(1)=\tilde{\Psi}_{y}(\tilde{M}-z I)(\tilde{M}-z I)^{-1} \pi(1)=\tilde{\Psi}_{y} \pi(1)=y$ is true for every $z \in \mathbf{C} \backslash E_{0}$, we conclude that the local spectrum $\sigma(V, y)$ is included in $E_{0}$. However, the relation $z_{0} \in\left(\mathbf{T} \backslash E_{0}\right) \cap \sigma(V, y)$ shows that this is impossible. Therefore, the vector $\varphi(V) y$ cannot be zero. Q.E.D.

Let $\rho: \mathbf{Z} \rightarrow[1, \infty)$ denote the mapping defined by $\rho(n):=\max \left(1,\left\|x_{n}\right\|\right), n \in \mathbf{Z}$. It is clear that $\rho(n+1) \leq K \rho(n)$ for every $n \in \mathbf{Z}$, where $K=\max (1,\|T\|)$. Let us consider the Banach space $A_{\rho}(\mathbf{T}):=\left\{f \in C(\mathbf{T}):\|f\|_{\rho}:=\sum_{n \in \mathbf{Z}}|\hat{f}(n)| \rho(n)<\infty\right\}$; clearly $A_{\rho}(\mathbf{T}) \subset A(\mathbf{T})$. The following lemma claims that, for every function $\varphi$ belonging to the restricted class $A_{\rho}(\mathbf{T})$, the operator $\varphi(V)$ transforms the vector $X x_{0}$ into the range of $X$.

Lemma 4. Given any function $\varphi \in A_{\rho}(\mathbf{T})$ we have $\varphi(V) y_{0} \in \operatorname{ran} X$, where $y_{0}:=$ $X x_{0}$.

Proof. Since $V^{n} X x_{0}=X x_{n}$ holds for every $n \in \mathbf{Z}$, it follows that $\varphi(V) y_{0}=$ $\sum_{n \in \mathbf{Z}} \hat{\varphi}(n) V^{n} X x_{0}=\sum_{n \in \mathbf{Z}} \hat{\varphi}(n) X x_{n}=\sum_{n \in \mathbf{Z}} X\left(\hat{\varphi}(n) x_{n}\right)$. In view of the fact that $\varphi \in A_{\rho}(\mathbf{T})$ we have $\sum_{n \in \mathbf{Z}}|\hat{\varphi}(n)|\left\|x_{n}\right\| \leq \sum_{n \in \mathbf{Z}}|\hat{\varphi}(n)| \rho(n)<\infty$, and so the series $\sum_{n \in \mathbf{Z}} \hat{\varphi}(n) x_{n}$ strongly converges to a vector $v \in \mathcal{X}$. Since the transformation $X$ is continuous we infer that $X v=\sum_{n \in \mathbf{Z}} X\left(\hat{\varphi}(n) x_{n}\right)$, that is $\varphi(V) y_{0}=X v \in \operatorname{ran} X$. Q.E.D.

The following lemma provides a sufficient condition for the subspace $\mathcal{X}(T, f)$ not to be the zero space.

Lemma 5. Let $f$ be an arbitrary function in $A(\mathbf{T})$. If $(\mathbf{T} \backslash \operatorname{supp} f) \cap \sigma\left(V, y_{0}\right) \neq \emptyset$, then $\mathcal{X}(T, f) \neq\{0\}$. 
Proof. There exists an open arc $\alpha \subset \mathbf{T} \backslash \operatorname{supp} f$ such that $\alpha \cap \sigma\left(V, y_{0}\right)$ is not empty; let us choose a point $z_{0} \in \alpha \cap \sigma\left(V, y_{0}\right)$. Then $\alpha$ and $z_{0}$ have the form $\alpha=$ $\left\{e^{i t}: \vartheta_{1}<t<\vartheta_{2}\right\}$ and $z_{0}=e^{i t_{0}}$, where $\vartheta_{1}<t_{0}<\vartheta_{2} \leq \vartheta_{1}+2 \pi$. Let us consider the arc $\alpha_{0}:=\left\{e^{i t}:-\eta<t<\eta\right\}$, where $\eta:=(1 / 4) \min \left(\vartheta_{2}-t_{0}, t_{0}-\vartheta_{1}\right)$. Since the $\operatorname{sum} \sum_{n \in \mathbf{Z}} \log \rho(n) /\left(1+n^{2}\right)$ is finite, by a result of A. Atzmon there exists a function $\varphi_{0} \in A_{\rho}(\mathbf{T})$ and a point $w_{0}=e^{i \tau_{0}} \in \alpha_{0}\left(-\eta<\tau_{0}<\eta\right)$ such that $\operatorname{supp} \varphi_{0} \subset \alpha_{0}$ and $\varphi_{0}\left(w_{0}\right) \neq 0$; see [2, Lemma XII. 5.2]. Then the function $\varphi(z):=\varphi_{0}\left(e^{i\left(\tau_{0}-t_{0}\right)} z\right)(z \in \mathbf{T})$ belongs to $A_{\rho}(\mathbf{T})$; furthermore, $\varphi\left(z_{0}\right)=\varphi_{0}\left(w_{0}\right) \neq 0$ and $\operatorname{supp} \varphi \subset \alpha$.

We infer by Lemma 4 that $\varphi(V) y_{0} \in \operatorname{ran} X$, that is there exists a vector $v \in \mathcal{X}$ such that $\varphi(V) y_{0}=X v$. Lemma 3 implies that $\varphi(V) y_{0} \neq 0$, and so $v \neq 0$. Finally, the relation $\operatorname{supp} \varphi \subset \alpha \subset \mathbf{T} \backslash \operatorname{supp} f$ yields that $f \varphi=0$, whence $f(V) \varphi(V)=0$ follows. We obtain that $f(V) X v=f(V) \varphi(V) y_{0}=0$ and so the non-zero vector $v$ belongs to the subspace $\mathcal{X}(T, f)$. Q.E.D.

Now we are ready to prove our Theorem.

Proof of the Theorem. We know that the spectral radius $r(T)$ of the operator $T$ occurring in the Theorem is positive. Let us consider the operator $T^{\prime}=r(T)^{-1} T$. It is clear that $r\left(T^{\prime}\right)=1$ and that $T^{\prime}$ belongs to the class $\mathcal{C}_{1} \cdot\left(p^{\prime}, \mathcal{X}\right)$, where $p^{\prime}(n)=$ $r(T)^{-n} p(n)(n \in \mathbf{N}), p^{\prime} \in \mathcal{P}$. Furthermore, we have $T^{\prime} x_{n}^{\prime}=x_{n+1}^{\prime}$ for the vectors $x_{n}^{\prime}=r(T)^{-n} x_{n}(n \in \mathbf{Z})$. Since Hlat $\left(T^{\prime}\right)=\operatorname{Hlat}(T)$ is obviously true, we can assume in the sequel that $r(T)=1$.

Let us consider the invertible isometry $V \in \mathcal{L}(\mathcal{Y})$ and the intertwining transformation $X \in \mathcal{L}(\mathcal{X}, \mathcal{Y})$ introduced earlier. We want to show first that the peripheral local spectrum $\sigma\left(V, y_{0}\right) \cap \mathbf{T}$ contains infinitely many points for the vector $y_{0}=X x_{0}$. Indeed, let us assume that $\sigma\left(V, y_{0}\right) \cap \mathbf{T}$ consists only of the finitely many points $\left\{\lambda_{j}\right\}_{j=1}^{k}$. Let us consider the cyclic subspace $\mathcal{Y}_{0}:=\vee_{n \geq 0} V^{n} y_{0}$ and the restriction $V_{0}:=V \mid \mathcal{Y}_{0}$. We know that $\sigma\left(V_{0}\right)=\sigma\left(V, y_{0}\right)$; see [8, Lemma 1.3.(iv)]. Since the spectrum of the isometry $V_{0}$ does not cover the unit disc, we infer that $\sigma\left(V_{0}\right)=\left\{\lambda_{j}\right\}_{j=1}^{k}$. The Riesz-Dunford functional calculus yields a decomposition $\mathcal{Y}_{0}=\sum_{j=1}^{k} \dot{+} \mathcal{Y}_{j}$ reducing for $V_{0}$ such that $\sigma\left(V_{0} \mid \mathcal{Y}_{j}\right)=\left\{\lambda_{j}\right\}$ for every $1 \leq j \leq k$. In virtue of Gelfand's theorem we conclude that $V_{0} \mid \mathcal{Y}_{j}=\lambda_{j} I_{\mathcal{Y}_{j}}(1 \leq j \leq k)$; see [10]. Therefore, $q\left(V_{0}\right)=0$ holds with the polynomial $q(z)=\prod_{j=1}^{k}\left(z-\lambda_{j}\right)$. Since $X q(T) x_{0}=q(V) X x_{0}=q\left(V_{0}\right) y_{0}=0$ it follows that $q(T) x_{0}=0$. Thus the vectors $\left\{x_{n}\right\}_{n \in \mathbf{N}}$ are contained in a finite dimensional subspace, which contradicts the assumption. Hence $\sigma\left(V, y_{0}\right)$ contains infinitely many points on the circle $\mathbf{T}$.

Let us take a sequence $\left\{\lambda_{n}\right\}_{n \in \mathbf{N}}$ of distinct points in $\sigma\left(V, y_{0}\right) \cap \mathbf{T}$ converging to a point $\lambda_{0}$. We can choose a sequence $\left\{\alpha_{n}\right\}_{n \in \mathbf{N}}$ of open $\operatorname{arcs}$ on $\mathbf{T}$ such that $\lambda_{n} \in \alpha_{n}$ and $\alpha_{n}^{-} \cap\left(\bigcup_{j \neq n} \alpha_{j}\right)^{-}=\emptyset$ hold, for every $n \in \mathbf{N}$. Given any $n \in \mathbf{N}$, let $\beta_{n}$ be an open arc containing $\lambda_{n}$ such that $\beta_{n}^{-} \subset \alpha_{n}$. By the normality of the function algebra $A(\mathbf{T})$, there exists a function $f_{n} \in A(\mathbf{T})$ such that $f_{n} \mid \mathbf{T} \backslash \alpha_{n}=1$ and $f_{n} \mid \beta_{n}^{-}=0$.

In view of Lemmas 1 and $5, \mathcal{X}_{n}:=\mathcal{X}\left(T, f_{n}\right)$ is a non-zero hyperinvariant subspace of $T$. Exploiting again the normality of $A(\mathbf{T})$, we can find a function $g_{n} \in A(\mathbf{T})$ such that $g_{n} \mid\left(\bigcup_{j \neq n} \alpha_{j}\right)^{-}=0$ and $g_{n} \mid \alpha_{n}^{-}=1$. Let us consider the subspace $\mathcal{X}_{n}^{\prime}:=$ $\mathcal{X}\left(T, g_{n}\right)$. Since $\operatorname{ker} f_{n} \cap \operatorname{ker} g_{n}=\emptyset$ we infer by Lemma 2(i) that $\mathcal{X}_{n} \cap \mathcal{X}_{n}^{\prime}=\{0\}$. Furthermore, for any $j \neq n$ we have $\operatorname{ker} f_{j} \subset \alpha_{j} \subset \operatorname{ker} g_{n}$. Thus ker $f_{j}$ is contained 
in the interior of ker $g_{n}$, and so the subspace $\mathcal{X}_{j}$ is included in $\mathcal{X}_{n}^{\prime}$ by Lemma 2(ii). Therefore, we obtain that $\mathcal{X}_{n} \cap\left(\bigvee_{j \neq n} \mathcal{X}_{j}\right) \subset \mathcal{X}_{n} \cap \mathcal{X}_{n}^{\prime}=\{0\}$.

By the Wiener-Ditkin Theorem (see [2, Theorem XII.5.3]) there exists a sequence $\left\{h_{k}\right\}_{k \in \mathbf{N}}$ in $A(\mathbf{T})$ converging to the function $h=\chi-\lambda_{0}$ such that each $h_{k}$ is zero on an open arc $\gamma_{k}$ containing $\lambda_{0}$. Given any $k \in \mathbf{N}$, there exists a positive integer $n_{k}$ such that for every $j \geq n_{k}$ the closure of $\alpha_{j}$ is included in $\gamma_{k}$. An application of Lemma 2(ii) results in that $\bigvee_{j \geq n_{k}} \mathcal{X}_{j} \subset \mathcal{X}_{k}^{\prime \prime}:=\mathcal{X}\left(T, h_{k}\right)$. Let us consider a vector $u \in \bigcap_{n \in \mathbf{N}}\left(\bigvee_{j \geq n} \mathcal{X}_{j}\right)$. Then $u \in \bigcap_{k \in \mathbf{N}} \mathcal{X}_{k}^{\prime \prime}$, and so $h_{k}(V) X u=0$ holds for every $k \in \mathbf{N}$. Taking into account that the operators $\left\{h_{k}(V)\right\}_{k \in \mathbf{N}}$ converge (in norm) to the operator $h(V)$ we obtain that $h(V) X u=0$. Since $X$ is injective, the equations $X\left(T-\lambda_{0} I\right) u=h(V) X u=0$ imply that $\left(T-\lambda_{0} I\right) u=0$. If $T$ has no eigenvalues on the circle $\mathbf{T}$, then $u$ must be zero. Thus we obtain that $\bigcap_{n \in \mathbf{N}}\left(\bigvee_{j \geq n} \mathcal{X}_{j}\right)=\{0\}$, and the proof of the Theorem is completed. Q.E.D.

Let us assume now that $T$ is of the larger class $\mathcal{C}_{*} .(p, \mathcal{X})(p \in \mathcal{P})$, and let $\mathcal{X}_{0}(T, p)$ denote the set of vectors $x$ such that the sequence $\left\{\left\|T^{n} x\right\| / p(n)\right\}_{n \in \mathbf{N}}$ almost converges to zero. We know that $\mathcal{X}_{0}(T, p)$ is a hyperinvariant subspace of $T$, which is different from $\mathcal{X}$. By $\left[7\right.$, Theorem 3] the quotient operator $\hat{T}:=T / \mathcal{X}_{0}(T, p)$ belongs to the class $\mathcal{C}_{1} \cdot\left(p, \hat{\mathcal{X}}:=\mathcal{X} / \mathcal{X}_{0}(T, p)\right)$, provided the sequence $\left\{r(T)^{n} / p(n)\right\}_{n \in \mathbf{N}}$ does not almost converge to zero. Thus we obtain the following consequence of the Theorem.

Corollary. Let $T \in \mathcal{L}(\mathcal{X})$ be an operator of class $\mathcal{C}_{*} \cdot(p, \mathcal{X}), p \in \mathcal{P}$, and suppose that the sequence $\left\{r(T)^{n} / p(n)\right\}_{n \in \mathbf{N}}$ does not almost converge to zero. Let us assume that there exists a sequence $\left\{x_{n}\right\}_{n \in \mathbf{Z}}$ in $\mathcal{X}$ such that the linear manifold spanned by the vectors $\left\{x_{n}\right\}_{n \in \mathbf{N}}$ is infinite dimensional and disjoint from $\mathcal{X}_{0}(p, T), T x_{n}=x_{n+1}$ holds for every $n \in \mathbf{Z}$, and

$$
\sum_{n \in \mathbf{Z}} \frac{\log ^{*}\left(r(T)^{-n}\left\|x_{n}\right\|\right)}{1+n^{2}}<\infty .
$$

Then there exists a sequence $\left\{\mathcal{X}_{n}\right\}_{n \in \mathbf{N}}$ of hyperinvariant subspaces of $T$, each of them properly containing $\mathcal{X}_{0}(T, p)$, such that

$$
\mathcal{X}_{n} \cap\left(\bigvee_{j \neq n} \mathcal{X}_{j}\right)=\mathcal{X}_{0}(T, p)
$$

holds for every $n \in \mathbf{N}$. Furthermore, if $\sigma_{p}\left(T^{\#}\right) \cap r(T) \mathbf{T}=\emptyset$, then

$$
\bigcap_{n \in \mathbf{N}}\left(\bigvee_{j \geq n} \mathcal{X}_{j}\right)=\mathcal{X}_{0}(T, p)
$$

Here $T^{\#}$ stands for the adjoint of the operator $T$ acting in the dual space $\mathcal{X}^{\#}$.

Proof. As in the proof of the Theorem, we can assume that $r(T)=1$. Let $\left\{\hat{\mathcal{X}}_{n}\right\}_{n \in \mathbf{N}}$ be a sequence of hyperinvariant subspaces of the quotient operator $\hat{T} \in \mathcal{C}_{1} \cdot(p, \hat{\mathcal{X}})$ resulting from the Theorem. Let $\pi: \mathcal{X} \rightarrow \hat{\mathcal{X}}$ denote the quotient mapping. It is easy to check that the subspaces $\mathcal{X}_{n}:=\pi^{-1}\left(\hat{\mathcal{X}}_{n}\right)(n \in \mathbf{N})$ are hyperinvariant for $T$, properly contain $\mathcal{X}_{0}(T, p)$, and $\mathcal{X}_{n} \cap\left(\bigvee_{j \neq n} \mathcal{X}_{j}\right)=\mathcal{X}_{0}(T, p)$ holds for every $n \in \mathbf{N}$.

It remains only to verify that the condition $\sigma_{p}\left(T^{\#}\right) \cap \mathbf{T}=\emptyset$ implies that $\sigma_{p}(\hat{T}) \cap$ $\mathbf{T}=\emptyset$. Indeed, let us assume that $\lambda \in \sigma_{p}(\hat{T}) \cap \mathbf{T}$. Let $V_{+} \in \mathcal{L}\left(\mathcal{Y}_{+}\right)$and $X \in$ $\mathcal{L}\left(\mathcal{X}, \mathcal{Y}_{+}\right)$be the isometry and intertwining operator, respectively, associated with 
$T$; see [7, Section 5]. Since $\operatorname{ker} X=\mathcal{X}_{0}(T, p)$, the quotient map $\hat{X}:=X / \mathcal{X}_{0}(T, p)$ is injective having a dense range, and intertwines the operators $\hat{T}$ and $V_{+}$. It follows that if $\pi(x)(x \in \mathcal{X})$ is a non-zero eigenvector of $\hat{T}$ corresponding to $\lambda$, then $V_{+} y=$ $\lambda y$ holds for the non-zero vector $y=\hat{X} \pi(x)$. Since $\lambda \in \mathbf{T}$ is an eigenvalue of the isometry $V_{+}$, we infer that $\lambda$ is an eigenvalue of its adjoint $V_{+}^{\#}$, as well. (In fact, the sequence $\left\{(n+1)^{-1} \sum_{j=0}^{n}\left(\bar{\lambda} V_{+}\right)^{j} u\right\}_{n \in \mathbf{N}}$ converges to zero, for every vector $u$ in the closure of $\operatorname{ran}\left(I-\bar{\lambda} V_{+}\right)=\operatorname{ran}\left(V_{+}-\lambda I\right)$. Thus $\operatorname{ker}\left(V_{+}-\lambda I\right) \cap\left(\operatorname{ran}\left(V_{+}-\lambda I\right)\right)^{-}=\{0\}$, and so the subspace $\operatorname{ker}\left(V_{+}^{\#}-\lambda I\right)=\left(\operatorname{ran}\left(V_{+}-\lambda I\right)\right)^{\perp}$ is not zero.) Then the relations $T^{\#} X^{\#}=X^{\#} V_{+}^{\#}$ and $\operatorname{ker} X^{\#}=\{0\}$ readily imply that $\lambda \in \sigma_{p}\left(T^{\#}\right)$, which was to be proved. Q.E.D.

We conclude this paper with some remarks.

Remarks. 1. The argument applied in the proof of the Corollary shows that $\sigma_{p}(T) \cap$ $r(T) \mathbf{T} \subset \sigma_{p}\left(T^{\#}\right) \cap r(T) \mathbf{T}$ is true, for every operator $T$ of class $\mathcal{C}_{1} .(p, \mathcal{X})(p \in \mathbf{N})$.

2. The mere existence of a proper hyperinvariant subspace can be derived from Atzmon's [1, Theorem 1.1.(b)] under more restrictive conditions on the behaviour of the sequence $\left\{x_{n}\right\}_{n \in \mathbf{Z}}$. Namely, let $T$ be an operator in the class $\mathcal{C}_{*} .(p, \mathcal{X})(p \in \mathcal{P})$, and let us assume, for the sake of simplicity, that $r(T)=1$. Considering the invertible extension $V \in \mathcal{L}(\mathcal{Y})$ of the isometry $V_{+}$occurring in the proof of the Corollary, we have the relation $T^{\#} X^{\#}=X^{\#} V^{\#}$. Let us choose a vector $\psi \in \mathcal{Y}^{\#}$ such that $\varphi:=X^{\#} \psi \neq 0$, and form the vectors $\varphi_{n}:=X^{\#} V^{\# n} \psi, n \in \mathbf{Z}$. The sequence $\left\{\varphi_{n}\right\}_{n \in \mathbf{Z}}$ is clearly bounded, and $T^{\#} \varphi_{n}=\varphi_{n+1}$ for every $n \in \mathbf{Z}$.

Let us assume yet that there exists a non-zero sequence $\left\{x_{n}\right\}_{n \in \mathbf{Z}}$ in $\mathcal{X}$ such that $T x_{n}=x_{n+1}(n \in \mathbf{Z})$ and $\left\{\left\|x_{n}\right\|\right\}_{n \in \mathbf{Z}}$ is dominated by a Beurling sequence $\rho: \mathbf{Z} \rightarrow[1, \infty)$. The latter condition means that $\rho(0)=1, \rho(m+n) \leq \rho(m) \rho(n)$ for every $m, n \in \mathbf{Z}, \sum_{n \in \mathbf{Z}} \log \rho(n) /\left(1+n^{2}\right)<\infty$, and there exists a positive constant $c$ such that $\left\|x_{n}\right\| \leq c \rho(n)$ holds for every $n \in \mathbf{Z}$. Under these assumptions Atzmon's theorem yields the existence of a proper hyperinvariant subspace, provided $T$ is not scalar multiple of the identity.

3. The condition $\rho(m+n) \leq \rho(m) \rho(n)$ actually means additional restriction. This can be seen from the following example. Let $\vartheta: \mathbf{Z} \rightarrow[1, \infty)$ be the sequence defined by $\vartheta(n):=\exp \left(4^{k}\right)$ if $n=k 2^{k}(k \in \mathbf{N})$, and let $\vartheta(n):=1$ otherwise. The sum $\sum_{n \in \mathbf{Z}} \log \vartheta(n) /\left(1+n^{2}\right)$ is clearly finite. Let us assume that a Beurling sequence $\rho$ dominates $\vartheta$, that is $\vartheta \leq c \rho$ with a positive constant $c$. Then, for every $k \in \mathbf{N}$, we have

$$
4^{k}=\log \vartheta\left(k 2^{k}\right) \leq \log c+\log \rho\left(k 2^{k}\right) \leq \log c+k 2^{k} \log \rho(1),
$$

which is clearly a contradiction.

\section{NOTE ADDED IN PROOF}

In the proof of Lemma 5 we have used Atzmon's lemma in its form appearing in Beauzamy's book. Professor Atzmon brought to our attention that this form is not correct, namely, the condition $\rho_{n+1} \geq c \rho_{n}(n \in \mathbf{Z}, c>0)$ is missing in the assumptions. However, instead of the Atzmon lemma we can refer to a recent result by Paul Koosis, where the previous condition is not assumed. The exact reference is the Corollary in the paper A local estimate, involving the least superharmonic majorant, for entire functions of exponential type, Algebra i Analiz. 10 (3), (1998), 45-64. We gratefully thank A. Atzmon for this correction. 


\section{REFERENCES}

[1] A. Atzmon, On the existence of hyperinvariant subspaces, J. Operator Theory, 11 (1984), 3-40. MR 85k:47005

[2] B. Beauzamy, Introduction to Operator Theory and Invariant Subspaces, North Holland, Amsterdam, 1988. MR 90d:47001

[3] I. Colojoara and C. Foias, Theory of generalized spectral operators, Gordon and Breach, New York, 1968. MR 52:15085

[4] R. G. Douglas, On extending commutative semigroups of isometries, Bull. London Math. Soc, 1 (1969), 157-159. MR 39:7458

[5] J.-P. Kahane, Séries de Fourier absolument convergentes, Springer-Verlag, 1970. MR 43:801

[6] Y. Katznelson, An introduction to harmonic analysis, Dover, New York, 1976. MR 54:10976

[7] L. Kérchy, Operators with regular norm-sequences, Acta Sci. Math. (Szeged), 63 (1997), 571-605. CMP 98:04

[8] L. Kérchy and J. van Neerven, Polynomially bounded operators whose spectrum on the unit circle has measure zero, Acta Sci. Math. (Szeged), 63 (1997), 551-562. CMP 98:04

[9] G. G. Lorentz, A contribution to the theory of divergent sequences, Acta Math., 80 (1948), 167-190. MR 10:367e

[10] J. Zemánek, On the Gelfand-Hille theorems, Banach Center Publications, Volume 30, Polish Academy of Sciences, Warszawa, 1994, 369-385. MR 95f:47009

Bolyai Institute, University of Szeged, Aradi vértanúk tere 1, H-6720 Szeged, HunGARY

E-mail address: kerchy@math.u-szeged.hu 